\title{
DSP-Based Stepping Motor Drivers for the LHC Collimators
}

\author{
A. Masi, G. Conte, R. Losito, and M. Martino
}

\begin{abstract}
The control electronics of the LHC collimators stepping motors will be located in radiation safe zones up to 800 meters far from the motors. With such cable lengths the standard chopping drivers do not work properly because of the voltage losses on the cable and even more because of the high cable capacitance. The capacitance in fact produces a ringing phenomenon on the driver feedback current that limits the control chopping frequency to the point of being incompatible with the tight EM emissions requirements of the LHC tunnel. In some cases the feedback loop may even become unstable and the driver would fail. The problem was solved by accurately modeling the overall motor-cable system taking into account non-linearities due to hysteresis and eddy currents and by designing an adaptive digital controller, self-tuning to the real cable length. The controller will aim at increasing the chopping frequency to reduce the spectral density of the emissions and at damping the oscillations of the feedback current to avoid instability. In this paper the model of the motor-cable system as well as the digital controller are described. Particular attention is devoted to the hardware implementation based on a TI Cx2000 DSP.
\end{abstract}

Index Terms-Current regulators, stepping motors drivers, TI Cx2000 DSP, transmission line.

\section{INTRODUCTION}

$\mathbf{T}$ HE LARGE Hadron Collider (LHC) will be installed in the existing $27 \mathrm{~km}$ circumference tunnel, about $100 \mathrm{~m}$ underground, previously housing the Large Electron Positron Collider (LEP). Its design is based on superconducting twin-aperture magnets which operate in a superfluid helium bath at $1.9 \mathrm{~K}$ [1].

The high luminosity to be provided by the LHC relies on storing, accelerating, and colliding beams with unprecedented intensities at energies of $14 \mathrm{TeV}$ in the center of mass. The transverse energy density of the nominal beam is 1000 times higher than in any other existing proton storage ring. Tiny fractions of the stored beam suffice to quench a superconducting LHC magnet or even to destroy parts of the accelerator. The energy stored in the two LHC beams ( $\sim 350 \mathrm{MJ})$ is sufficient to melt almost 1 ton of copper.

In order to protect the machine components, in particular the superconducting magnets, from uncontrolled beam losses more than 100 collimators will be installed all around the $27 \mathrm{~km}$ long

Manuscript received May 10, 2007; revised October 3, 2007.

A. Masi, G. Conte, and R. Losito are with the CERN, Geneva 1211, Switzerland (e-mail: alessandro.masi@ cern.ch).

M. Martino is with Dipartimento di Scienze Fisiche, the Università degli Studi di Napoli Federico II, Napoli 80126, Italy.

Color versions of one or more of the figures in this paper are available online at http://ieeexplore.ieee.org.

Digital Object Identifier 10.1109/TNS.2007.913475

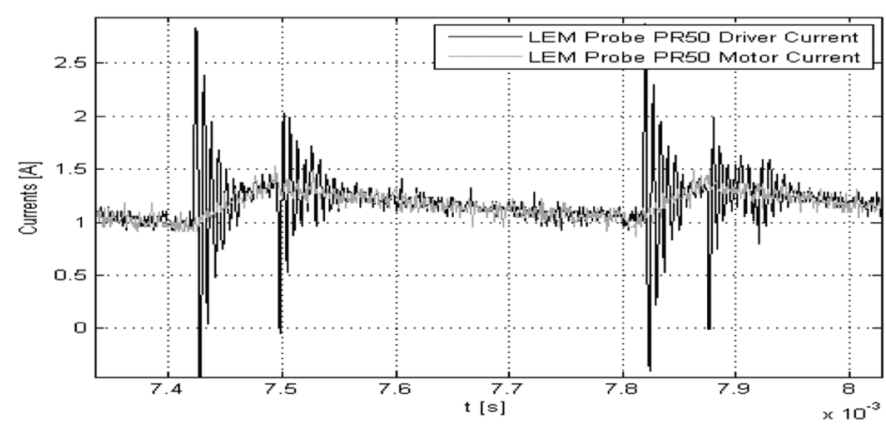

Fig. 1. The ringing effect. Difference between the real current in the motor (in grey) and the current measured at the driver output (in black) that is used to control the current in the motor. This measurement was taken with 280 meters of cable between the motor and the driver.

accelerator. The collimators will also clean the beams from excessive background for the experiments by scraping away the unwanted particles with 1.2 meter long blocks (hereafter referred to as "jaws") of different materials, such as graphite, fiber-reinforced carbon, copper or tungsten, disposed coherently with the concept of multistage collimation [2]. Each collimator consists of one or two jaws that have to be positioned with very high accuracy $( \pm 10 \mu \mathrm{m})$ close to the beam in order to absorb energetic particles. Each jaw is moved by two stepping motors, in order to ensure good repeatability of the position during parallel and skew movement of the jaws. Each collimator has therefore four stepping motors to be controlled; some collimators also have a fifth motor, to adjust the vertical position of the jaws with respect to the beam trajectory, to use a different part of the absorbing block in case of damage from the beam.

Since the stepping motors will be installed in a radioactive area, their power supply unit cannot be placed close to them.

Thus, the drivers of the collimators stepping motors will have to cope with cables ranging from 100 meters up to 800 meters. At the chopping frequency normally used for the control ( $\sim 18 \mathrm{kHz}$ ), and due their lengths, these cables behave as transmission lines. In particular the high capacitance seen by the drivers brings to instability (ringing) of the feedback current.

An example of ringing phenomenon on the phase feedback current (measured by the driver) is shown in Fig. 1.

It can be seen that the feedback current becomes dramatically different from the real current flowing in the correspondent motor phase. In all the commercial stepping motor drivers based on current control that we have tested, the control algorithm fails with a consequent degradation of the positioning performances. ElectroMagnetic Emissions are also enhanced by the presence of current oscillations. 


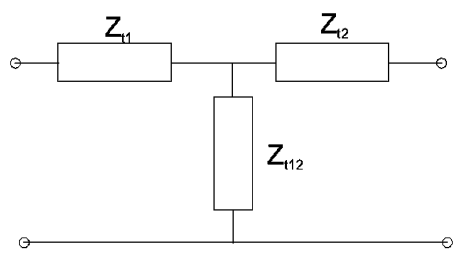

Fig. 2. Equivalent two port lumped T-net model for a twisted pair.

In order to address these problems, we conducted a thorough study on cable characteristics, motor modeling and designed a digital controller based on a TI Cx2000 DSP to address at the same time the control problem and the EM emissions. In this last respect, the strategy was to move as high as possible the chopping frequency in order to reduce the spectral density of the emitted energy. In the LHC, the most sensitive equipment close to the collimator electronics are the Beam Loss Monitors that have a bandwidth of $500 \mathrm{kHz}$.

\section{Characterization OF THE Motor-CABle System}

\section{A. The Cable}

The cable used to connect the driver to the motor is a twisted pair line and has to be modeled, in the range of lengths and frequencies of interest, as a transmission line with losses.

Interactions with the other twisted pairs in the same cables are neglected. We verified experimentally that this assumption is correct to our purposes. Fig. 2 shows an equivalent lumped two port T- net model [4].

The three impedances can be modeled as follows [3]:

$$
\begin{aligned}
Z_{t 1} & =Z_{t 2}=Z_{0}\left[\operatorname{coth}\left(\gamma_{0} h\right)-\operatorname{csch}\left(\gamma_{0} h\right)\right] \\
Z_{t 12} & =\operatorname{csch}\left(\gamma_{0} h\right)
\end{aligned}
$$

where

$$
\gamma_{0}=\sqrt{(r+j 2 \pi f l)(g+j 2 \pi f c)}
$$

is the propagation coefficient,

$$
Z_{0}=\sqrt{\frac{r+j 2 \pi f l}{g+j 2 \pi f c}}
$$

is the characteristic impedance and $h$ is the length of the line. $r, l, g$ and $c$ are the resistance, inductance, conductance and capacitance per unit length and are functions of the frequency. In the frequency range of interest for the control algorithm we can assume them constant.

When a load impedance $Z_{L}$ is connected to the line as shown in Fig. 3, the input impedance seen by the driver is:

$$
Z_{\text {in }}=Z_{0} \frac{Z_{L}+Z_{0} \tanh \left(\gamma_{0} h\right)}{Z_{0}+Z_{L} \tanh \left(\gamma_{0} h\right)}
$$

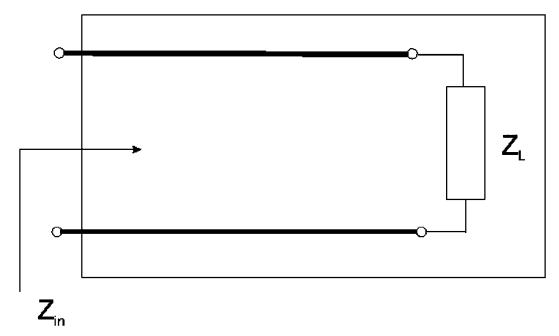

Fig. 3. Input line impedance (one phase): the impedance seen by the driver.
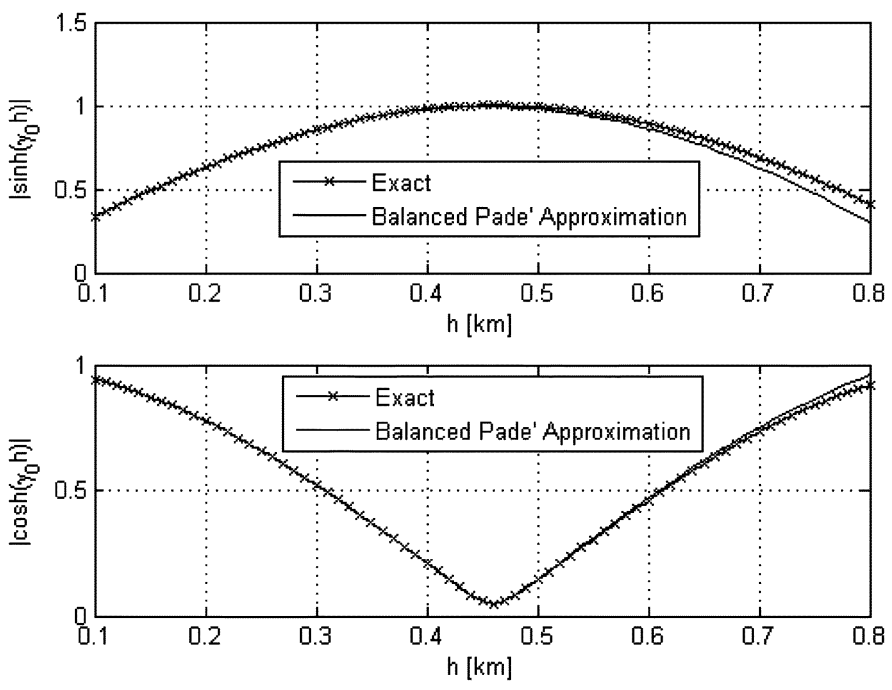

Fig. 4. Hyperbolic functions approximation using 4th order balanced Padè approximants. Plots refer to a frequency of $100 \mathrm{kHz}$.

Combining the previous expressions, one can rewrite (4) in the Laplace domain as follows:

$Z_{\text {in }}=\sqrt{\frac{r+s l}{g+s c}} \frac{Z_{L} \sqrt{g+s c} \cosh \left(\gamma_{0} h\right)+\sqrt{r+s l} \sinh \left(\gamma_{0} h\right)}{\sqrt{r+s l} \cosh \left(\gamma_{0} h\right)+Z_{L} \sqrt{g+s c} \sinh \left(\gamma_{0} h\right)}$.

Hyperbolic terms can conveniently be approximated by 4 th order balanced Padé approximants [5], [6], up to length of $800 \mathrm{~m}$ and frequencies up to $100 \mathrm{kHz}$, as shown in Fig. 4

$$
\begin{aligned}
& \sinh \left(\gamma_{0} h\right) \cong \frac{\gamma_{0} h+0.1142\left(\gamma_{0} h\right)^{3}}{1-0.0525\left(\gamma_{0} h\right)^{2}+0.0014\left(\gamma_{0} h\right)^{4}} \\
& \cosh \left(\gamma_{0} h\right) \cong \frac{1+0.4475\left(\gamma_{0} h\right)^{2}+0.0168\left(\gamma_{0} h\right)^{4}}{1-0.0525\left(\gamma_{0} h\right)^{2}+0.0014\left(\gamma_{0} h\right)^{4}}
\end{aligned}
$$

From this 4th order development, one obtains a stable rational expression of (5). The approximated transfer function $Z_{\text {in }}$ is a 5 th order rational transfer function:

$$
Z_{\text {in }}(s)=\frac{p_{0}+p_{1} s+p_{2} s^{2}+p_{3} s^{3}+p_{4} s^{4}+p_{5} s^{5}}{q_{0}+q_{1} s+q_{2} s^{2}+q_{3} s^{3}+q_{4} s^{4}+q_{5} s^{5}}
$$

where $p_{i}$ and $q_{i}$ depend on the length of the cable. 

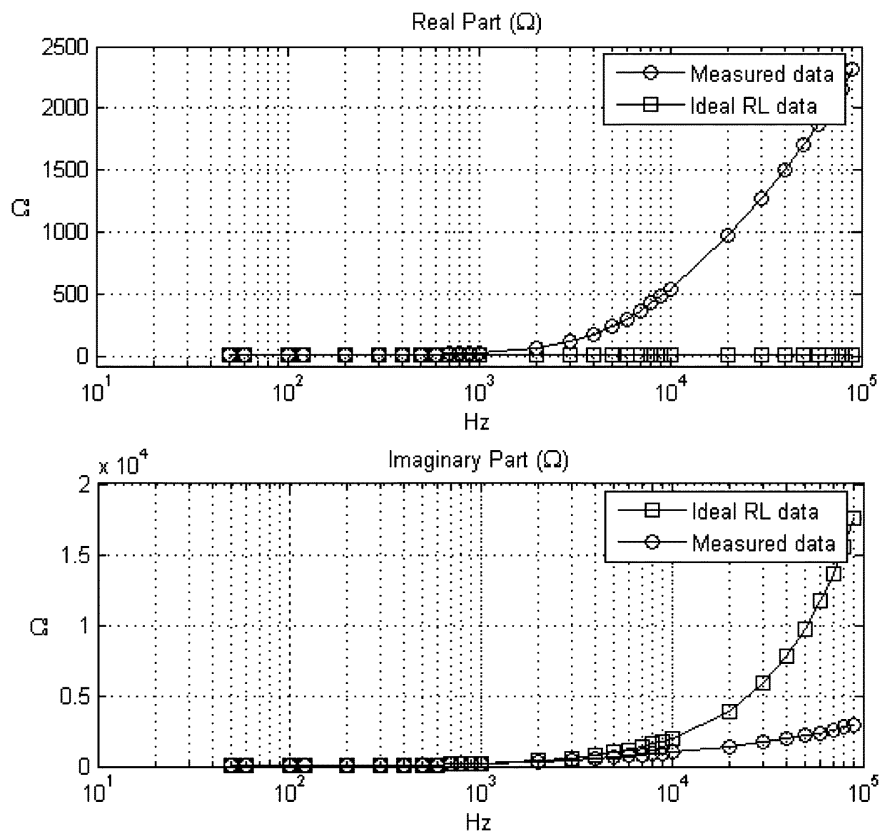

Fig. 5. Comparison between measured and RL ideal impedances.

\section{B. The Motor}

The motors used in the LHC collimation project are twophase hybrid stepping motors with 200 physical steps per revolution.

Impedance measurements over a wide frequency range have been performed, using an RLC Meter (Fluke PM 6306), in order to find an accurate electrical model. An RL model based on datasheet values fails already above $1 \mathrm{kHz}$, as shown in Fig. 5 .

It is important to note that the real part, in particular, is not constant with frequency, and this is due to the increasing losses induced in the iron core of the motor by eddy currents.

These losses can be modeled by adding the shunt impedances $R_{f e}$ and $L_{f e}$ as in the equivalent circuit of Fig. 6, which represents one motor phase.

$R_{f e}$ takes into account the real power dissipated by eddy currents and $L_{f e}$ takes into account the reactive power stored for magnetic hysteresis. Both $R_{f e}$ and $L_{f e}$ are functions of frequency.

The input impedance of the circuit in Fig. 6 can be written as (8) shown at the bottom of the page.

Measurement data have been fitted to an analytical expression for $R_{f e}(f)$ and $L_{f e}(f)$ :

$$
\begin{aligned}
R_{f e}(f) & =[354.3 \ln (0.03564 f)+0.03564 f] \Omega \\
L_{f e}(f) & =\left(70.58 f^{-0.765}\right) H \\
L_{w} & =0.03001 H .
\end{aligned}
$$

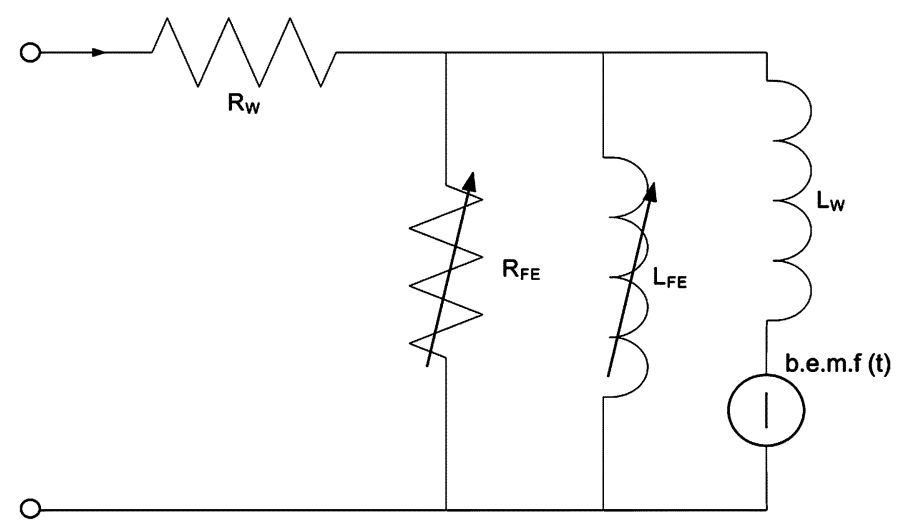

Fig. 6. Equivalent motor phase electrical circuit, including iron losses.b.e.m. $\mathrm{f}(\mathrm{t})$ is the back electromotive force.
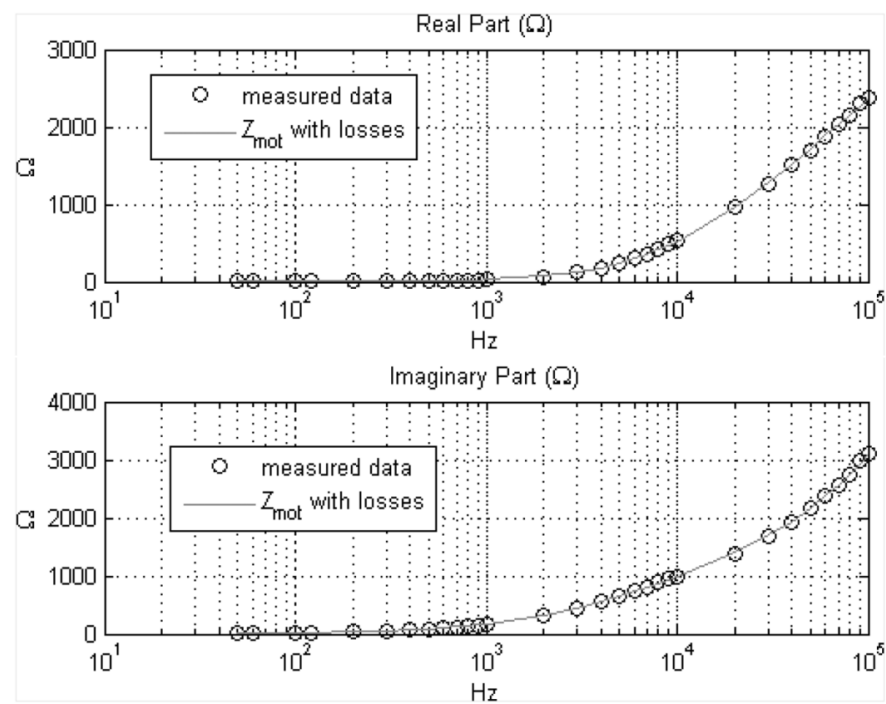

Fig. 7. Comparison between measured data and motor model with losses.

A new expression for $Z_{L}$ (in the Laplace domain) can now be considered:

$$
Z_{L}(s)=\frac{R_{w}+\left[L_{\mathrm{eq}}+R_{w} \tau_{\mathrm{eq}}\right] s}{1+s \tau_{\mathrm{eq}}} \approx \frac{R_{w}+L_{\mathrm{eq}} s}{1+s \tau_{\mathrm{eq}}}
$$

where the last expression is obtained considering that $L_{\mathrm{eq}}+$ $R_{w} \tau_{\mathrm{eq}}=\left(1+R_{w} / R_{f e}\right) L_{\mathrm{eq}}$ and that $R_{f e} \gg R_{w}$.

Model (10) matches satisfactorily the measured data as it can be seen in Fig. 7.

Finally, a complete model of the motor has to take into account also the back Electro-Motive Force (EMF), that can be modeled as a voltage generator placed in series to the motor windings $L_{w}$.

$$
Z_{L}(f)=\frac{L_{w} L_{f e}(f)\left[R_{w}+R_{f e}(f)\right] j 2 \pi f+R_{w} R_{f e}(f)\left[L_{w}+L_{f e}(f)\right]}{L_{w} L_{f e}(f) j 2 \pi f+R_{f e}(f)\left[L_{w}+L_{f e}(f)\right]} .
$$




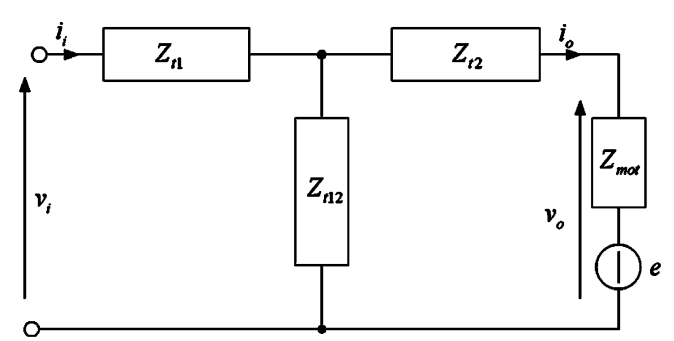

Fig. 8. Equivalent circuit of cable-motor phase.

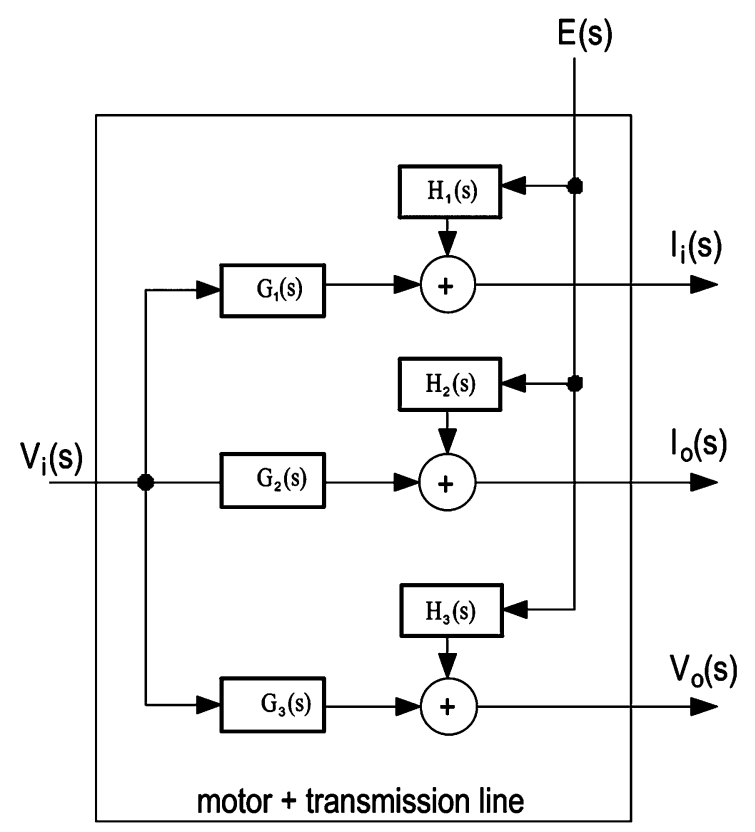

Fig. 9. Block scheme describing cable and motor phase.

\section{The Model of the Motor-Cable System}

Fig. 8 shows the circuit describing a motor phase with the cable. $Z_{\text {mot }}$ and $e$ represent the Thevenin equivalent impedance and back EMF of the motor.

$v_{o}$ and $i_{o}$ are the electrical quantities acting on the motor and can be seen as the output variables of the model. $v_{i}$ is the voltage supplied to the line by the driver and represents the control variable of the digital controller. Finally, $i_{i}$ is the measurable variable to be used for the feedback.

Operating directly in the Laplace domain it follows:

$$
\begin{aligned}
I_{i}(s) & =\frac{V_{i}(s)}{Z_{\text {in }}(s)}=G_{1}(s) V_{i}(s) \\
I_{o}(s) & =G_{2}(s) V_{i}(s) \\
V_{o}(s) & =G_{3}(s) V_{i}(s) \\
I_{i}(s) & =H_{1}(s) E(s) \\
I_{o}(s) & =H_{2}(s) E(s) \\
V_{o}(s) & =H_{3}(s) E(s) .
\end{aligned}
$$

The above equations can be represented graphically as in Fig. 9.

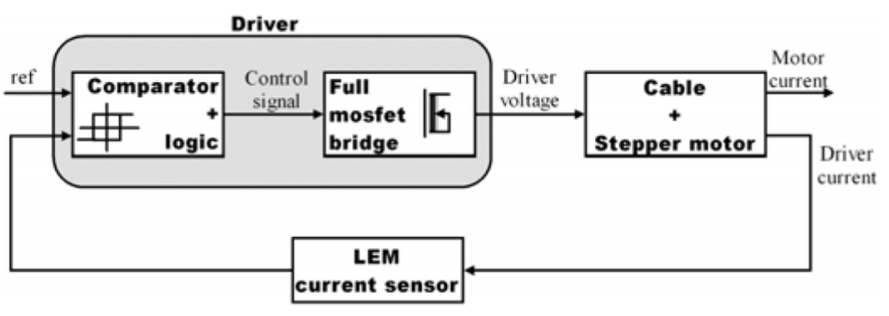

Fig. 10. Driver block scheme.

The six transfer functions defined in (11)-(16) have the following expressions:

$$
\begin{aligned}
G_{1}(s) & =\frac{Z_{0} \cosh \left(\gamma_{0} h\right)+Z_{\mathrm{mot}} \sinh \left(\gamma_{0} h\right)}{Z_{0}\left[Z_{\text {mot }} \cosh \left(\gamma_{0} h\right)+Z_{0} \sinh \left(\gamma_{0} h\right)\right]} \\
G_{2}(s) & =\frac{1}{Z_{\text {mot }} \cosh \left(\gamma_{0} h\right)+Z_{0} \sinh \left(\gamma_{0} h\right)} \\
G_{3}(s) & =\frac{Z_{\mathrm{mot}}}{Z_{\mathrm{mot}} \cosh \left(\gamma_{0} h\right)+Z_{0} \sinh \left(\gamma_{0} h\right)} \\
H_{1}(s) & =-\frac{1}{Z_{\mathrm{mot}} \cosh \left(\gamma_{0} h\right)+Z_{0} \sinh \left(\gamma_{0} h\right)} \\
H_{2}(s) & =-\frac{\cosh \left(\gamma_{0} h\right)}{Z_{\mathrm{mot}} \cosh \left(\gamma_{0} h\right)+Z_{0} \sinh \left(\gamma_{0} h\right)} \\
H_{3}(s) & =-\frac{Z_{\mathrm{mot}} \cosh \left(\gamma_{0} h\right)}{Z_{\mathrm{mot}} \cosh \left(\gamma_{0} h\right)+Z_{0} \sinh \left(\gamma_{0} h\right)}
\end{aligned}
$$

The Padè approximants (6) are finally used to get rational transfer functions of (17)-(22).

\section{Model VALIDATION}

The model has been tested comparing simulations with experimental results measured on a commercial standard stepping motor driver based on a hysteresis current regulator (see Fig. 10). No correction to the ringing is applied yet.

Fig. 11 shows a comparison between simulations and real data. The cable length for this case is very small $(<1$ meter) to validate separately the model of the motor. The reference signal has been generated in order to realize a microstepping control with 2000 steps/revolution and a step rate of 400 steps/second. The commercial driver used works with a minimum $T_{\text {on }}$ algorithm [7] at a chopping frequency of $18 \mathrm{kHz}$, using a single slow discharge path in a full bridge configuration. Measured and simulated data fit perfectly.

A second verification (see Fig. 12) has been done connecting a 280 meter long cable and performing a full step control (200 step/rev). Current ringing, in this case, obliges to decrease the chopping frequency down to $2.5 \mathrm{kHz}$ to obtain stable operation, since the feedback current can be measured only once the ringing is damped after each voltage pulse of the chopping driver.

\section{Digital Controller Design}

\section{A. The De-Ringing Filter}

The ringing effect experienced on the driver current (the one to be used for the feedback) is due to the cable shunt impedance (see Fig. 8).The current on the motor side of the long cable is 


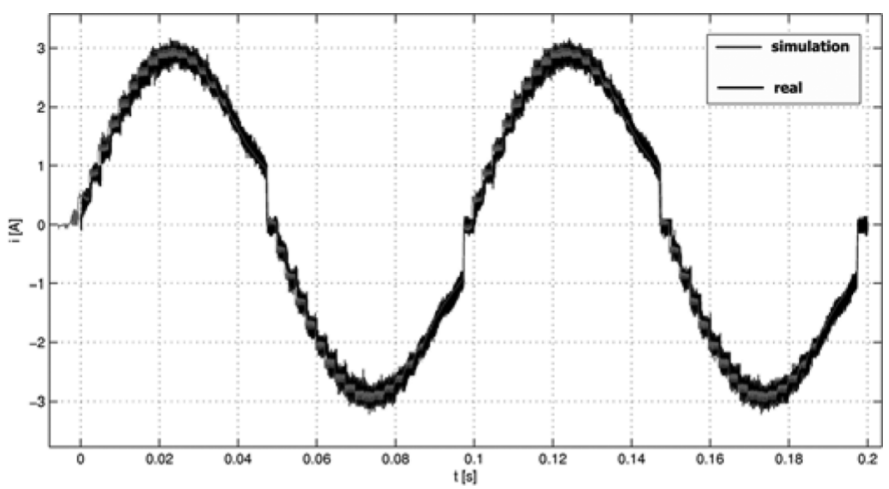

Fig. 11. Comparison between the simulated (grey) process and the real (black) data measurement (no cable). The analysis has been done using microstepping resolution (2000 steps/revolution) and a step rate of $400 \mathrm{steps} / \mathrm{s}$.

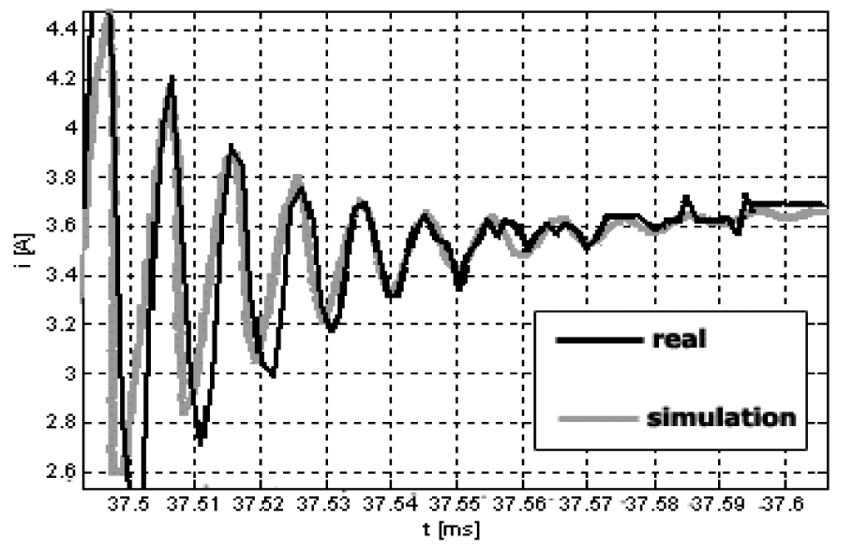

Fig. 12. Simulated (grey) vs measured (black) feedback current with $280 \mathrm{~m}$ cable length. The extinguishing time from simulation matches perfectly the measured one. $\tau_{\mathrm{eq}}$ and $L_{\mathrm{eq}}$ have been evaluated at $2.5 \mathrm{kHz}$.

not affected by the ringing because it is smoothed by the motor inductance. To correctly control the motor phase current is then necessary to find an analytical relationship between the two currents on the opposite side of the long cable. This can be achieved by filtering out the ringing.

The transfer function of this filter (that we named "the estimator" $G_{\text {est }}$ ) is in fact:

$$
G_{\mathrm{est}}=\frac{I_{o}}{I_{i}}=\frac{Z_{t 12}}{\left(Z_{t 2}+Z_{t 12}+Z_{\mathrm{mot}}\right)}
$$

Equation (23) can be simplified as follows:

$$
G_{\mathrm{est}}=\frac{Z_{0}}{Z_{0} \cosh \left(\gamma_{0} h\right)+Z_{\mathrm{mot}} \sinh \left(\gamma_{0} h\right)} .
$$

This expression has to be transformed in the $Z$ domain to be implemented as a digital filter.

We used the Tustin approximation [8]:

$$
G_{\mathrm{estd}}(z)=\left.G_{\mathrm{est}}(s)\right|_{s=\frac{2}{T_{s f}}} \frac{z-1}{z+1}
$$
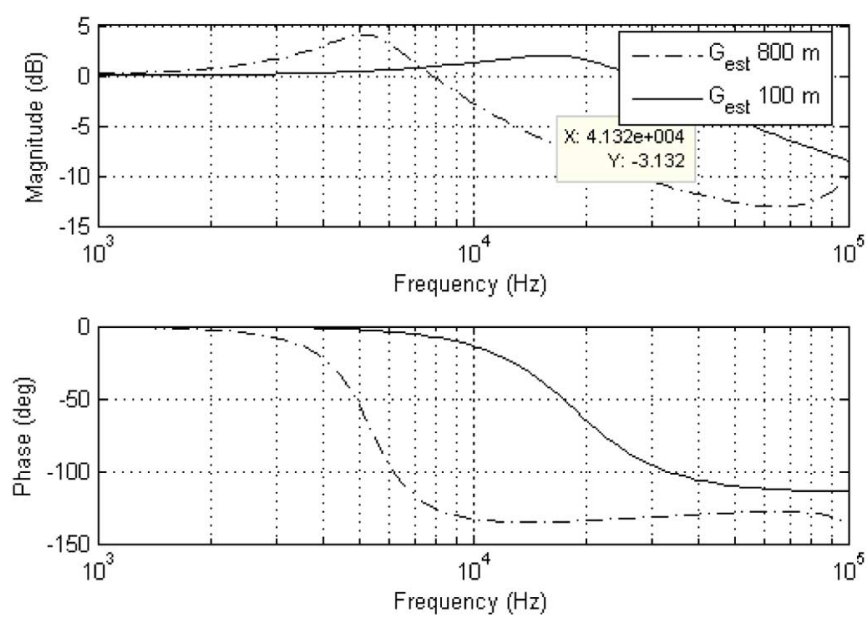

Fig. 13. The frequency response of $G_{\text {est }}$ (s).

where $T_{s f}=1 / f_{s f}$ is the sampling period used for the filter and has to be chosen in order to respect the Nyquist-Shannon criterion'. Simulations show that, in the worst case $(h=100 \mathrm{~m})$, $B_{3 \mathrm{~dB}} \approx 41 \mathrm{kHz}$ (Fig. 13). We chose $f_{s f}=300 \mathrm{kHz}$.

Using again the Padè approximants $(5)$, a 5 th order rational expression is obtained.

The digital filter has the following form:

$$
G_{\mathrm{estd}}(z)=\frac{n_{0}+n_{1} z+n_{2} z^{2}+n_{3} z^{3}+n_{4} z^{4}+n_{5} z^{5}}{1+d_{1} z+d_{2} z^{2}+d_{3} z^{3}+d_{4} z^{4}+d_{5} z^{5}}
$$

where $n_{i}$ and $d_{i}$ depend on the cable length $h$.

\section{B. The Controller}

The design of the controller is performed in the time domain, using the measured frequency data response [9].

The regulator proposed has an integrator to assure static precision, a pole, a zero and a suitable gain to have good dynamical performances:

$$
R(s)=\mu \frac{1+s \tau_{z}}{s\left(1+s \tau_{p}\right)} .
$$

The controller parameters

$$
\left[\begin{array}{lll}
\mu & \tau_{z} & \tau_{p}
\end{array}\right]
$$

have been calculated for several different cable length values in order to guarantee, in each case, a rise time $T_{r} \leq 500 \mu$ s and an overshoot $S_{\%} \leq 15 \%$, leading to the following dynamical parameters [9]:

$$
\begin{aligned}
\varphi_{m} & =75.4^{\circ} \\
\omega_{c} & =6.66 \mathrm{krad} / \mathrm{s} \\
\left|F\left(j \omega_{c}\right)\right| & =33.8 \mathrm{~dB} .
\end{aligned}
$$

${ }^{1}$ Exact reconstruction of a continuous-time baseband signal from its samples is possible if the signal is bandlimited and the sampling frequency is greater than twice the signal bandwidth. 

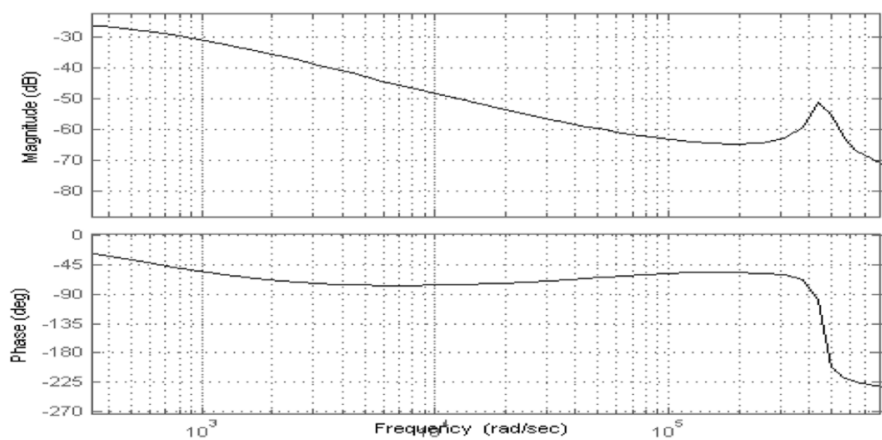

Fig. 14. Bode diagram of $G_{2}(s)$. The analysis is done using the measured motor frequency data and the transmission line model of the cable of Fig. 2. The analysis is done in the worst case $(800 \mathrm{~m})$.
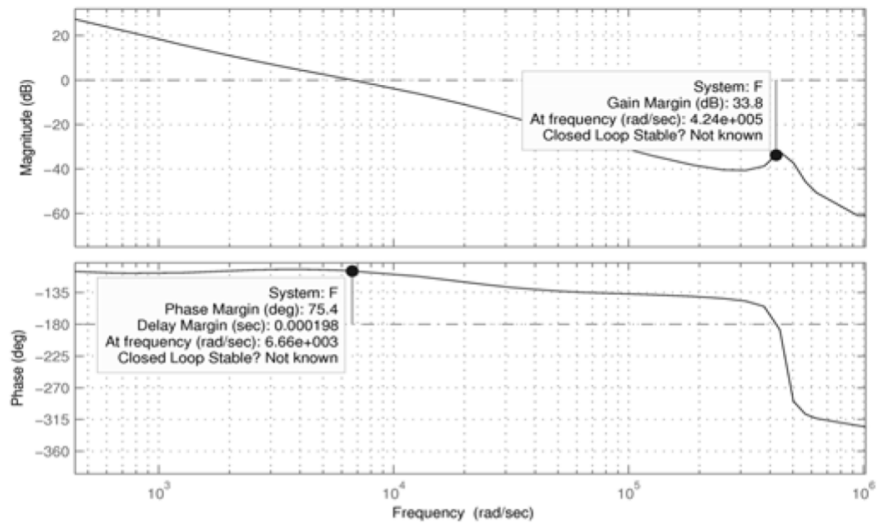

Fig. 15. Bode diagram of $R(s) G_{2}(s)(h=800 \mathrm{~m})$.

The analysis has been conducted on fifteen discrete cases with the cable length defined as $h_{k}=(100+50 k) \mathrm{m}, k=0 \ldots 14$.

A matrix can thus be created and used to load the specific controller with the parameters corresponding to the actual cable length.

The analysis in the worst case of dynamic performances $(h=$ $800 \mathrm{~m})$ is here proposed. Fig. 14 shows the Bode diagram of $G_{2}(s)$ when $h=800 \mathrm{~m}$.

The calculated parameters (28), in this case are:

$$
\left[\begin{array}{lll}
210 \cdot 10^{3} & 8.62 \cdot 10^{-4} & 4.69 \cdot 10^{-5}
\end{array}\right]
$$

The resulting function satisfies the specified dynamic parameters defined in (29), as shown in Fig. 15.

The controller (27) has to be discretized in order to obtain a digital version that can be implemented on a Digital Signal Processor (DSP).

Firstly, the sampling frequency of the controller (different from the sampling time of the filter previously mentioned) is chosen according to the heuristic rule:

$$
\alpha \omega_{c} \leq \omega_{s c} \leq 10 \alpha \omega_{c}
$$

where $\omega_{c}$ is the critical pulse of the control system and $5 \leq$ $\alpha \leq 10$. In this case, since $\omega_{c}=6.66 \cdot 10^{3} \mathrm{rad} / \mathrm{s}$, choosing $\omega_{s c} \approx 30 \omega_{c}$ it results:

$$
\omega_{s c}=199.8 \mathrm{krad} / \mathrm{s} \Rightarrow f_{s c} \approx 30 \mathrm{kHz}
$$

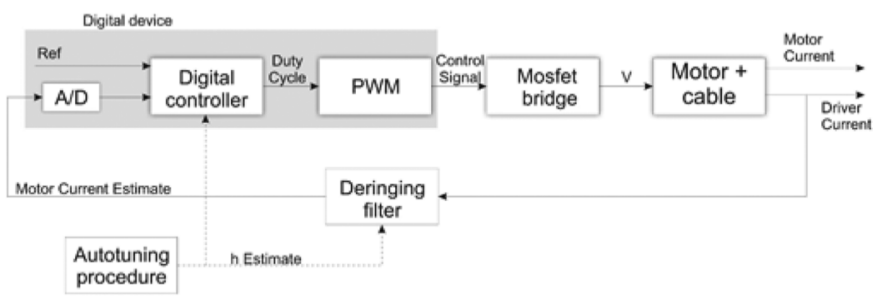

Fig. 16. Digital driver block scheme.

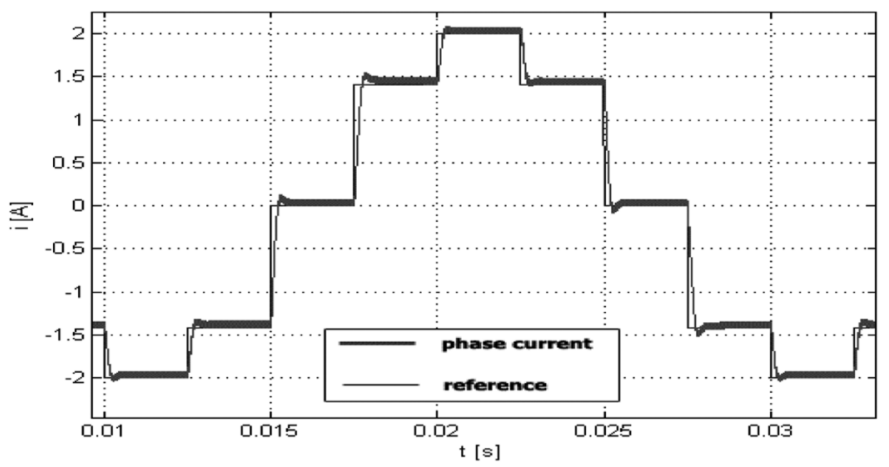

Fig. 17. Half Mode, 200 steps/s. Phase current.

The bilinear transformation method [8] has been used to obtain the expression of the discrete time controller. For $h=800$ $\mathrm{m}$, for instance we have, in the discrete time domain:

$$
\begin{aligned}
R_{d}(z) & =\left.R(s)\right|_{s=\left(2 / T_{s c}\right)(z-1 / z+1)} \\
& =50.3 \frac{z^{2}+1.906 z-48.39}{z^{2}-1.477 z+0.4767} .
\end{aligned}
$$

\section{Simulation Results}

Fig. 16 shows the block scheme describing the digital drive implementation. The digital controller output is modulated with a Pulse Width Modulator (PWM) that controls the mosfet bridge in order to perform both a fast and a slow discharge in the off phase.

The minimum chopping frequency that can be chosen is $f_{c}=$ $f_{s c}=30 \mathrm{kHz}$.

An autotuning procedure, consisting in the measurement of the resistance of the cable + motor system, is executed only at start-up to determine the length of the cable and therefore to choose the right set of coefficients to be used by the de-ringing filter.

The scheme in Fig. 16 has been implemented in Simulink ${ }^{\mathrm{TM}}$ and a simulation in half step mode is shown in Fig. 17. The result shows that the current in the motor is now well reproduced at the entrance of the controller, and an accurate regulation of the output current can be performed.

\section{THE HARDWARE IMPLEMENTATION}

\section{A. The Experimental Setup}

The control algorithm has been implemented on a TMS320F2812A DSP chip of the TI Cx2000 family.

In this experiment, we used an $\mathrm{eZdsp}^{\mathrm{TM}} \mathrm{F} 2812$ stand-alone DSP board as a hardware platform. This allows full-speed veri- 


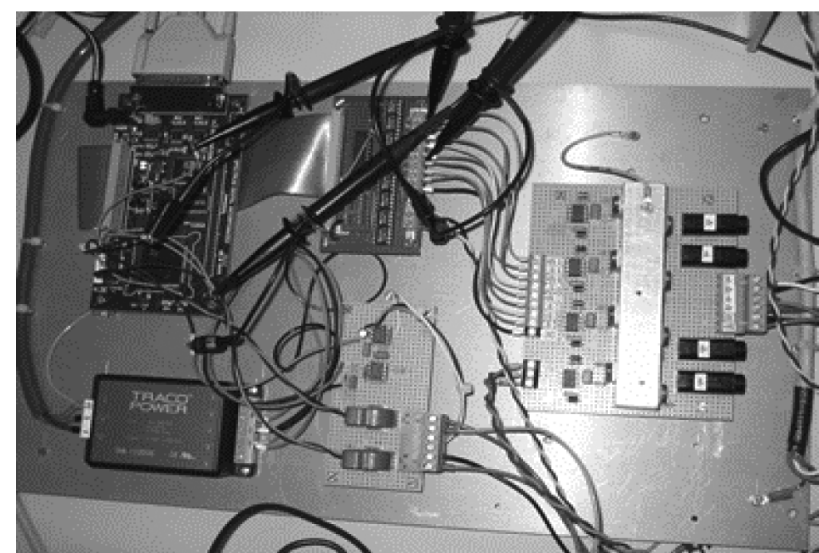

Fig. 18. Digital driver prototype.

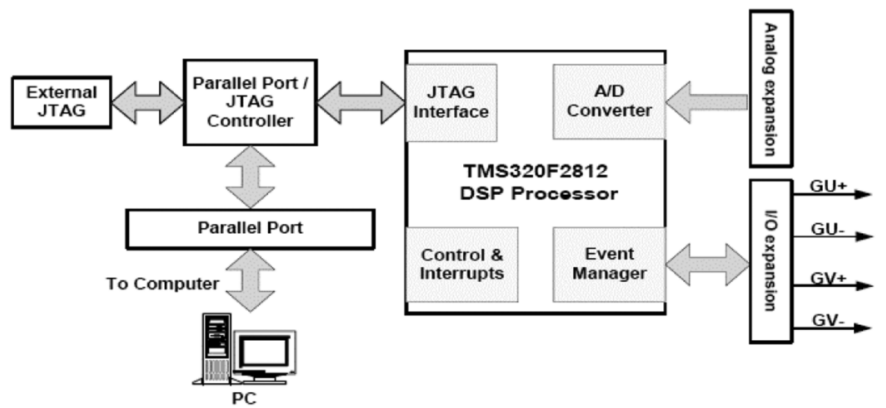

Fig. 19. eZdsp F2812 DSP board block diagram.

fication of the high-level language code built on Code Composer Studio ${ }^{\mathrm{TM}}$ tools.

The hardware prototype (Fig. 18) for this experiment is composed by the following sections:

- Single-board DSP (eZdsp F2812 stand-alone DSP board);

- Buffer stage composed by buffer amplifiers of the 74HCT family, optocoupled with a 6 N137;

- Full mosfet bridge composed by IR2181 mosfet drivers and IR537 mosfets;

- Current sensors LEM LTSR 6;

- Stepper motor (Maccon SM 87.2.18M2N);

- 135 V Power supply.

The DSP chosen has several useful peripherals including twelve pulse width modulators (PWM), sixteen channels of 12-bits analog-to-digital converters, and two quadrature encoder input modules (QEP).

On the evaluation board two expansion connectors, digital I/O and analog I/O, provide additional interfacing capabilities with other circuits. The PWM output pins in the I/O expansion are used in this hardware experiment for the mosfet bridge PWM control. Fig. 19 shows a block diagram of the eZdsp ${ }^{\mathrm{TM}}$ F2812 DSP board.

Among various function modules of the TMS320F2812A, the Event Manager (EV) module is used to program the PWM control algorithms. The EV includes two identical blocks, EVA and EVB. Both come with 16-bits general-purpose timers, compare units, and capture units. Each PWM output bit is used to control one mosfet. Fig. 20 highlights the EV function blocks and the control outputs.

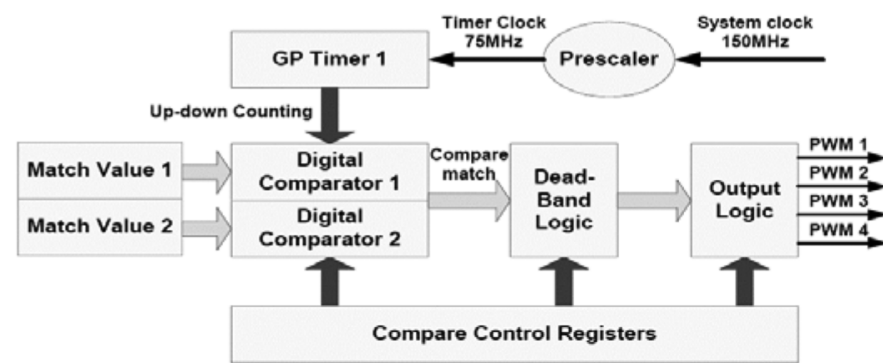

Fig. 20. Utilized EVA function block diagram.

EVA block with four PWM outputs PWM1 to PWM4 can satisfy the mosfets control requirements.

\section{B. The RT Software Architecture}

1) Cable Length Estimate: A simple algorithm has been written and implemented on the DSP in order to get an estimate of the cable length. The easiest way to get a measure of $h$ (cable length), is to perform a steady state current measurement. A DC measurement is not possible with the supply voltage of the full bridge without damaging the motor. A PWM approach has been used to limit the motor current. Feeding the motor-cable with a constant duty cycle modulated voltage signal, the phase current (neglecting the mosfets voltage drop) will reach a mean steady state value defined by:

$$
i_{d c}=\frac{V_{d c} \cdot \text { Dutyc }+(1-\text { Dutyc }) \cdot V_{\mathrm{off}}}{R_{w}+r h} \approx \frac{V_{d c} \cdot \text { Dutyc }}{R_{w}+r h}
$$

where the above simplification has been done considering that $V_{\text {off }}$ (the diode voltage drop) is at most $-1.3 \mathrm{~V}$ and it is negligible with $V_{d c}$ around $135 \mathrm{~V}$ (the DC supply voltage used in our case, chosen to guarantee a sufficient working voltage at the motor even with $800 \mathrm{~m}$ cable). The current will have a ripple due to the switching. Anyway, an accurate measure of $i_{d c}$ can be obtained filtering the current signal with a low-pass filter. The lowpass bandwidth has to be chosen sufficiently small to neutralise high frequency effects.

Choosing it around $10 \mathrm{~Hz}$ will require about 0.5 seconds to stabilise the result.

The cable length estimate is found with the following formula:

$$
\tilde{h}=\frac{1}{r}\left(\frac{V_{d c} \cdot \text { Dutyc }}{\tilde{i}_{d c}}-R_{w}\right)
$$

where $r$ is the nominal cable resistance per unit length.

Fig. 21 shows a blocks scheme describing the procedure to estimate the cable length $h$. Then the value is used to generate the de-ringing filter coefficients $n_{i}(h), d_{i}(h)$ and the controller parameters $\left[\mu(h) \tau_{z}(h) \tau_{p}(h)\right]$.

2) Tasks Architecture: The RT software architecture has to implement:

- the current reference generator;

- the de-ringing filter;

- the controller.

The tasks architecture is shown in Fig. 22. Interrupts are used to schedule the tasks. These are generated by internal timers for 


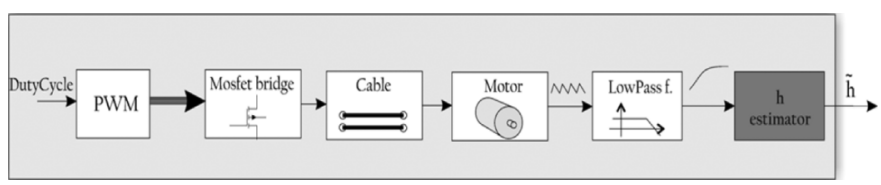

Fig. 21. Cable length estimation blocks scheme. The Self-tuning algorithm has to run at start-up to select the right parameters for the controller.

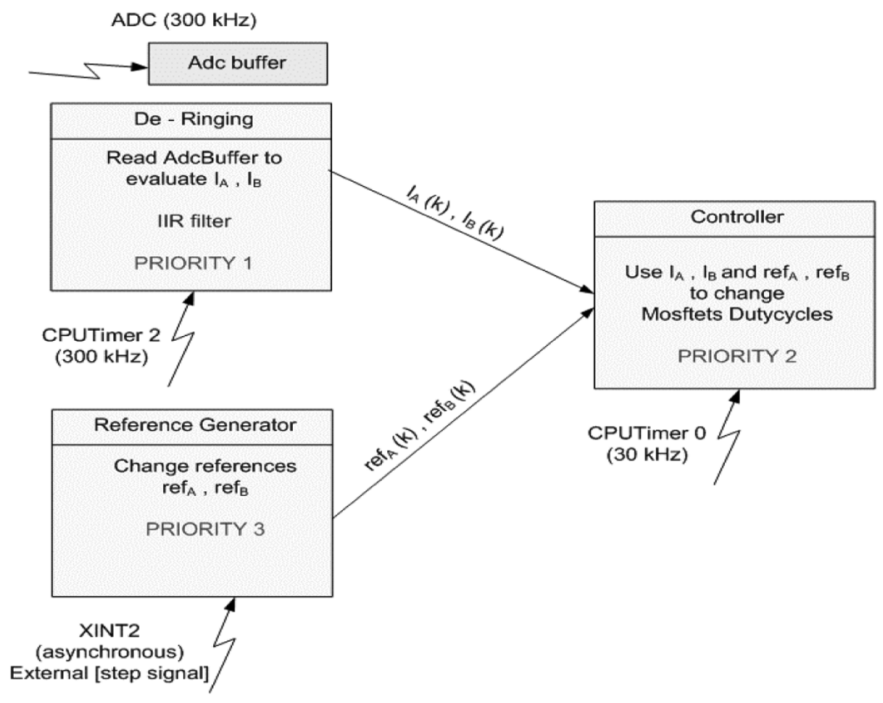

Fig. 22. Tasks Architecture.

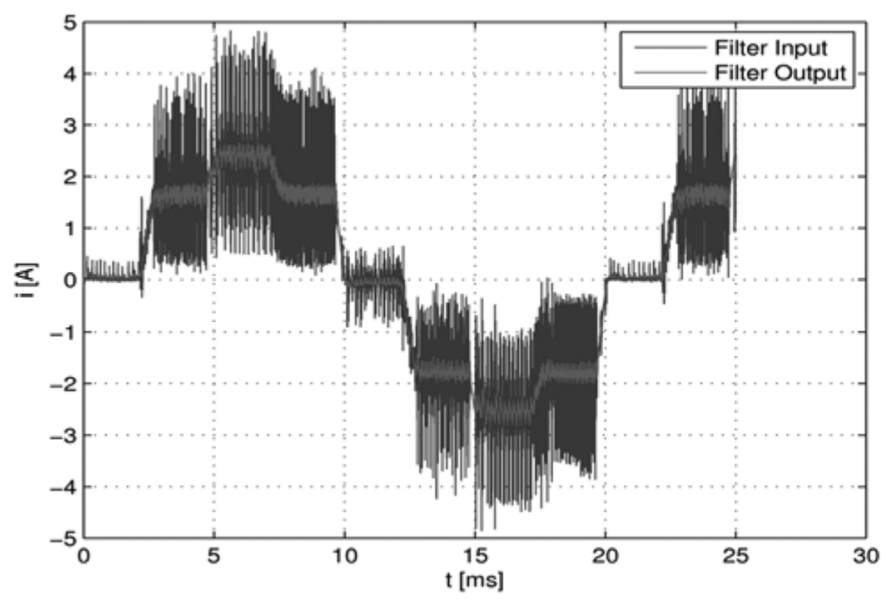

Fig. 23. Estimated motor current (de-ringing output, in grey) vs driver current (in black). $800 \mathrm{~m}$ cable length-halfstep-1 turn/s.

the de-ringing filter and the controller periodic tasks. The reference generator, instead, is triggered by the step signal coming on an external interrupt pin of the DSP.

\section{Experimental Results}

The output of the de-ringing filter compared with the input current is shown in Fig. 23. The ringing is completely eliminated.

Fig. 24, finally, shows a measurement of the filter output, ringing free, and compares it with the real motor phase current.

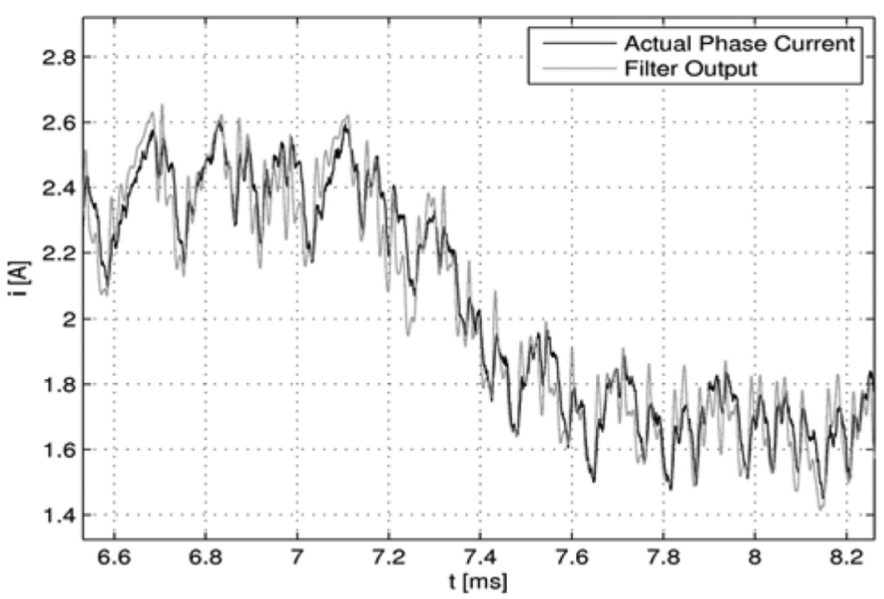

Fig. 24. Estimated vs actual phase current- $800 \mathrm{~m}$ cable length-halfstep-1 turn/s.

The two curves are satisfactorily identical, the error in current is only a few percent.

\section{CONCLUSION}

An adaptive digital controller to drive the LHC collimators motors has been designed starting from an accurate model identification of the cable-motor system. The digital filter tuned on the cable length has eliminated the problem of ringing on the feedback current ensuring a stable and accurate control of the motor up to cable lengths up to $800 \mathrm{~m}$. The controller has been implemented on the TMS320F2812A DSP.

The preliminary measurements on the prototype show an improvement in the EM noise emitted on the cable.

\section{ACKNOWLEDGMENT}

The authors would like to thank their colleagues of the AB/ATB/LPE Section, in particular J. Lendaro, B. Simon, and C. Mitifiot for the hardware implementation of the prototype and C. Serpico for EMC measurements.

\section{REFERENCES}

[1] O. Brüning, P. Collier, P. Lebrun, S. Myers, R. Ostojic, J. Poole, and P. Proudlock, "The LHC main ring, LHC design report," CERN-2004003, 2004.

[2] R. Assmann et al., "The final collimation system for the LHC," presented at the Euro. Particle Accelerator Conf. (EPAC), Edinburgh, Scotland, U.K., 2006.

[3] F. Pierre-Gerard, Systémes de Télécommunications, 1st ed. Lausanne, Switzerland: Edition Georgi, 1999.

[4] G. Miamo and A. Maffucci, Transmission Lines and Lumped Circuits, 1st ed. San Diego, CA: Academic, 2001.

[5] S. Lin and E. S. Kuh, "Transient simulation of lossy interconnects based on the recursive convolution formulation," IEEE Trans. Circuits Syst., Fundam. Theory Appl., vol. 39, no. 11, pp. 879-892, Nov. 1992.

[6] S. P. Singh, J. H. W. Burry, and B. Watson, Approximation Theory and Spline Functions, vol. 136, NATO ASI Series. Mathematical and Physical Sciences, 1984.

[7] STMicroelectronics, Geneva, Switzerland, "Fully integrated two phase stepper motor driver," L6208 AN 1451 Appl. Note, 2003.

[8] K. J. Åström and W. Björn, Computer-Controlled Systems: Theory and Design, III ed. Englewood Cliffs, NJ: Prentice-Hall, 1997.

[9] P. Bolzern, R. Scattolini, and N. Schiavoni, Fondamenti di Controlli Automatici, 2nd ed. Milan, Italy: McGraw Hill, 2004. 